\title{
Letter to the editor: "Revisiting the role of vitamin D levels in the prevention of COVID-19 infection and mortality in European countries post infections peak"
}

\author{
Dimitra Rafailia Bakaloudi ${ }^{1} \cdot$ Michail Chourdakis $^{1}$ (I)
}

Received: 3 June 2021 / Accepted: 27 August 2021 / Published online: 16 September 2021

(c) The Author(s), under exclusive licence to Springer Nature Switzerland AG 2021

\section{Dear Editor,}

On July 2020, an interesting article entitled "The role of vitamin $D$ in the prevention of coronavirus disease 2019 infection and mortality by Ilie et al. [1], was published in Aging Clinical and Experimental Research. One month later, on August 2020, another relevant paper entitled "Revisiting the role of vitamin D levels in the prevention of COVID-19 infection and mortality in European countries post infections peak" by Singh et al. was also published from Aging Clinical and Experimental Research [2]. After careful examination of the methodological approach and the evidence used in both published articles, we found some inaccuracies and methodological failures which might have influenced the result of these studies. In addition, Maruotti et al. referred to further methodological inaccuracies of the same article [3].

First, we believe that both studies used data which were gathered too early in the COVID-19 pandemic. In particular, data about COVID-19 infections and mortalities for European countries were collected early May (8th and 12th May 2020), whereas COVID-19 was declared as a pandemic on March 11th, 2020; therefore, evidence regarding the outspread pattern of COVID-19 were not clear enough.

Moreover, both articles used the mean values of vitamin D concentration in European countries, to examine the correlation between vitamin $\mathrm{D}$ concentration and number of COVID-19 infections and mortalities. However, a mean value cannot be representative for a whole country since it is influenced by outliers and skewed populations. As one can read, data regarding Vitamin D concentration in different

Michail Chourdakis

mhourd@gapps.auth.gr

1 Laboratory of Hygiene, Social and Preventive Medicine and Medical Statistics, School of Medicine, Faculty of Health Sciences, Aristotle University of Thessaloniki, University Campus, 54124 Thessaloniki, Greece
European countries were obtained by Lips et al. 2019 [4]. Nevertheless, some of the data used included both children and adult population (i.e., data for UK), were not means but medians (i.e., data from Switzerland), included specific population subgroups (i.e., Slovakia: only women) or were rather old (i.e., Switzerland: data from 1992, Turkey: data from 2007) and therefore could not be used as representative for the year 2020 (See Supplementary Material). Moreover, some data seemed not to be obtained for Lips et al. study and the source of this information is missing (i.e., data from Italy, Spain, Hungary, Estonia and Sweden).

Recently, we have conducted the same analysis using updated data regarding both the COVID-19 and the mean Vitamin D concentration of each European country and significant correlation was not found for infections $(r=-0.245$; $p=0.378)$ or mortality rates $(r=-0.362 ; p=0.185)$ (See Supplementary Material).

We believe that a more accurate analysis would have been succeeded if the authors had used the prevalence of vitamin $\mathrm{D}$ deficiency for each country instead of the mean values. This kind of analysis was performed in our paper [5] and statistically significant correlation was found only for mortality rates. We understand that the use of "prevalence of vitamin D deficiency" is not an ideal one, but it can be more representative than a mean value. Well-designed randomized controlled trials are needed to better determine/define the role of Vitamin D in COVID-19 infection.

Results from these 2 studies [1,2], should be cautiously used or extrapolated as they could direct towards to the use of vitamin D as a preventive measure of COVID-19 infection in specific countries. However, such an approach cannot be considered as appropriate, taking into account the result of our analysis, and information from a recent editorial in LANCET, in which the limited evidence for the role of vita$\min \mathrm{D}$ as a preventive measure in COVID-19 is underlined [6]. 
Yours sincerely.

Supplementary Information The online version contains supplementary material available at https://doi.org/10.1007/s40520-021-01975-z.

\section{Declarations}

Conflict of interest On behalf of all authors, the corresponding author states that there is no conflict of interest.

Statement of human and animal rights Not applicable.

Informed consent Not applicable.

\section{References}

1. Ilie PC, Stefanescu S, Smith L (2020) The role of vitamin D in the prevention of coronavirus disease 2019 infection and mortality. Aging Clin Exp Res 32:1195-1198

2. Singh S, Kaur R, Singh RK (2020) Revisiting the role of vitamin $\mathrm{D}$ levels in the prevention of COVID-19 infection and mortality in European countries post infections peak. Aging Clin Exp Res 32:1609-1612

3. Maruotti A, Belloc F, Nicita A (2020) Comments on: The role of vitamin D in the prevention of coronavirus disease 2019 infection and mortality. Aging Clin Exp Res 32:1621-1623

4. Lips P, Cashman KD, Lamberg-Allardt C et al (2019) Current vitamin D status in European and Middle East countries and strategies to prevent vitamin D deficiency: a position statement of the European Calcified Tissue Society. Eur J Endocrinol 180:P23-P54

5. Bakaloudi DR, Chourdakis M (2021) A critical update on the role of mild and serious vitamin D deficiency prevalence and the COVID-19 epidemic in Europe. Nutrition 93:111441. https://doi. org/10.1016/j.nut.2021.111441

6. The Lancet Diabetes and Endocrinology (2021) Vitamin D and COVID-19: why the controversy? Lancet Diabetes Endocrinol 9:53

Publisher's Note Springer Nature remains neutral with regard to jurisdictional claims in published maps and institutional affiliations. 